\title{
EDITORIAL:
}

\section{Technology and Health Care Official Journal of the European Society for Engineering and Medicine: A New Journal}

\author{
Peter F. Niederer
}

The creation of a new scientific Journal requires careful consideration and a number of solid reasons. Excess of printed information is already a problem and the need for a journal which covers the overlapping areas of Technology and Health Care, respectively, is not a priori obvious, although such a journal cannot readily be found to date.

New Journals today are often the consequence of increasing specialisation or the advent of new scientific fields and the concurrent intention of a number of highly specialised scientists to have a suitable source of in-depth information. As the title of this new Journal, Technology and Health Care however suggests, its scope is in contrast rather broad and general. The reasons for this are as follows.

When the 4th Medical and Health Research Programme of the EC was approaching its end two questions arose which were intimately related to its success. First, numerous individual researchers active in the many fruitful networks established in the course of the Concerted Actions of this Programme were anxious to keep their networks in existence, at least in some loose form, and approached COMAC-BME, the supervising Committee, with respect to possible ways to reach this goal. Second, it became apparent that many of the results and insights obtained during the Programme could be of benefit to many people active in health care in general, including persons way beyond the group of researchers involved in a Concerted Action. A need therefore existed to create an organization involving doctors, engineers, scientists, practitioners, hospital administrators, members of health authorities, etc., with a professional basis in medical technology.

A further consideration was associated with the growing integration and self-confidence of Europe. A need was felt to increase the visibility of Europe's biomedical community and to strengthen the European presence in the increasingly competitive world.

As a result, the European Society for Engineering and Medicine was founded during the 1st European Conference on Biomedical Engineering in Nice, France in February 1991. The president of this Society, Prof. J.E.W. Beneken, outlines its intentions and aims in his article The European Society for Engineering and Medicine fills a need in Europe in this first issue. In view of these aims and the considerations mentioned above, a scientific journal wholly for the Society with a firm basis in Europe, having a broad scope and which addresses itself both to the Medical as well as to 
the Biomedical Engineering worlds is a natural and essential outgrowth associated with the activities of the Society.

The question then immediately arises why an existing journal could not be identified which could have been adapted to the objectives of the Society. Several obvious advantages would have been gained with such a procedure of which the Board of ESEM was well aware. However, financial reasons and/or too much specialisation of possible candidate journals impeded such a solution. The opportunity was therefore taken to create a new journal and to structure it from scratch according to the goals and intentions of the Society. As such, it is expected to emphasize the future of medical technology and the interrelation between medicine and biomedical engineering and support mutual stimulation. Significant developments which are taking place in the industrialized world and which will strongly influence the impact of technology on health care are thereby to be taken into account:

First, rapid scientific and technological progress seems to cause an increasing separation between the medical and technical world. To find the right position between the two extremes of (1) the specialist who finally knows everything about nothing and of (2) the generalist who ends up with knowing nothing about everything is more difficult than ever. The need to present a complex medical or technical subject for a more general, nevertheless qualified readership without compromising scientific rigor is increasing, difficult and not fulfilled to date.

Second, the health care system is experiencing an enormous cost increase. The total expenditure for health care in the US as reported by NIH was $\$ 817$ billion for 1991 . This is in agreement with figures from Europe, e.g., Switzerland for which it was SFr 27 billion (equivalent ot about US\$20 billion) with an approximately fourty times smaller population. The cost is thereby growing rapidly. While in the US an increasing part of the population is no longer able to participate in the health system for financial reasons, the Federal Government of Switzerland had to put emergency law procedures into action in order to prevent such a development. In either case, the political implica- tions are severe and there is no reason to believe that these problems will rapidly be solved in the future. Cost seems in contrast to be rising as if there were unlimited sources and it can therefore be anticipated that this aspect will be one of the main issues in health care in the future years. Accordingly, questions relating to efficacy of medical technology, quality control, needs and meaningful application of technology in health care will become of primary importance. It will be a primary task of biomedical engineering to make use of advanced technology to develop diagnostic and therapeutic devices and procedures which are more specific, safer and easier to use, less traumatic for the patient, assist in reducing the length of an eventual hospitalization or enable an ambulant treatment and which are less expensive and require less maintenance and operational expense in spite of superior performance; for example minimally invasive surgery and diagnostics is an important topic in this context.

The almost explosive growth of computer hardand software power stimulates new developments and improvements of medical technology in general. Moreover, medical micro- and nanotechnology extends more and more into the cellular and molecular level. The possibilities, e.g., of genetic repair are no longer science fiction but become reality and are in part based on high-tech procedures. The biomedical engineer of the future, already now exposed to a rapidly changing pattern of an enormously extended interdisciplinary environment, will have to cope with cellular and molecular technologies including, in addition, bioprocess engineering. Likewise, micromechanical devices are expected to open new possibilities in the treatment of diseases.

Excess in the application of medical technology in the form of extending life unnecessarily in obviously hopeless situations has raised criticism in the past. Further improved capabilities and sophistication of devices and medical technology could increase the tendency of undertaking unjustified, even inhumane attempts to extend life. Another aspect which is to some extent connected with this issue is related to the increasing proportion of aged persons of our society. Achievements of BME which can be of help in keeping the aged 
independent and capable of continuing their usual life are of great importance.

Yet another development of the industrialized society is associated with its increasing aversion to animal experiments and at the same time increasing interest in non-technical, "traditional" medicine. Replacement of animal experiments by unproblematic models including the development of models based on virtual reality is an important task in this context.

In the future, professionals based in biomedical engineering, regardless of whether their background be from the medical, engineering or administrative side, cannot keep themselves out of these highly controversial issues. The fact that Biomedical Ethics was expressly added to the new programme of the EC in the area of biomedicine, BIOMED 1 ( $c f$. the note of Dr. V. Thévenin referring to this programme in this issue) as an own field of research emphasizes this point.

According to the description of the Journal, it is intended to cover the "overlapping areas between Physics, Engineering, Informatics and $\mathrm{Hu}-$ man Biology, Basic Medical Sciences and Clinical Sciences" which is an ambitious goal and raises high expectations. It is thought to serve as a forum for the presentation of new concepts, procedures and devices, furthermore, for the discussion of questions relating to the application of technology in health care in general, to efficiency, cost effectiveness and quality of technical medicine and to society-related questions. Besides scientific contributions of a high level, tutorials on complex technical or medical problems and discussion papers are expected to be given special attention. Controversial issues will be presented and discussed in depth. All relevant aspects are thereby to be approached from a science- and technologyrelated point-of-view, and as such the journal covers all areas where technology and medicine, resp. health care have common problems, needs and interests. From the medical side all disciplines including nonclinical specialities are involved, while in turn from a more technology-oriented point-of-view the following topics are addressed (as is outlined in a concise form in the Aims and Scope of the journal):

- medical technology in general
- biomedical engineering (BME) in basic physiological and medical sciences

- BME in biotechnology, bioprocess engineering, cellular and molecular technology, genetic engineering

- medical micro- and nanotechnology/micromechanical devices

- drug delivery systems

- imaging techniques (ultrasound, MR, CT, PET, EIT, thermography, image analysis)

- bioelectronics

- electrograms (ECG, EEG, EMG, EOG)

- biomagnetism (SQUIDS, MCG, MEG)

- biosensors (chemical, optical, biological)

- life support systems/patient monitoring (ventilators, heart-lung machines, automatization)

- biocompatibility

- artificial organs/implants (skin, vessels, heart, pacemakers, defibrillators, kidney, pancreas, joints, ligaments, cochlea/hearing impairment)

- biomechanics (tissue characteristics, motion analysis, implant mechanics, trauma)

- biofluidmechanics

- medical informatics/systems analysis (knowledge-based systems, hospital and patient information systems, PACS, systems approach, statistics)

- mathematical modelling/simulation (virtual reality)

- neuroinformatics

- medical physics (X-ray, radiation therapy, dosimetry, physical therapy)

- medical optics and laser technology (photo dynamics, biostimulation, fluorescence, spectro scopy)

- BME in minimally invasive surgery, endoscopy

- microscopy (light, scanning, electron, tunnel, atomic force, associated image technology)

- lithotripsy

- hyperthermia

- telemetry

- clinical laboratory/laboratory automation (automated cytology, laboratory technology)

- rehabilitation/BME for aged persons

- efficacy and quality of diagnostic and therapeutic procedures

- general society-related and health policy-related topics (medical technology assessment, 
cost of medical equipment, regulations/test procedures, medical equipment data banks)

- biomedical ethics

- problems relating to animal experiments

- education in BME.

The journal publishes peer-reviewed:

- original scientific contributions,

- short communications,

- discussion papers and controversies,

- review articles and tutorials (upon invitation).

This inaugural issue is intended to demonstrate on the one hand the wide spectrum which will be addressed by the Journal and on the other hand the sequence of articles is to be viewed under the aspect of the application of technology in particularly important areas. Thereby, the authors attempt to treat a given problem in-depth rather than outlining the entire field comprehensively and thereby loosing scientific significance. The contributions are devoted to the disorders of the brain, their diagnosis and investigation and to rehabilitation which is of increasing importance in view of keeping handicapped persons as independent as possible; to diseases of the skeleton, in particular arthritis, which are still partially unsolved biological problems with enourmous social implications; to diseases of the heart which are likewise of great socio-economic importance and face at the same time fascinating perspectives due to the continuing improvement of mechanical assistance; to minimally invasive surgery which will be essential in reducing hospital expenditures and represents a demanding challenge for the biomedical engineer. Finally, the potential of knowledgebased systems will inevitably have enourmous consequences upon the utilization of medical expertise in general. To treat the latter topic, a novel approach was taken in that all relevant aspects are presented as discussions in the form of a written mini-symposium. This form of presentation is intended to be used for the discussion of topics whenever it deems appropriate, in particular for controversial issues.

It will be for the reader to decide whether this issue has reached the goal which it has set itself. Likewise, it will be the readers and the future authors of the Journal who determine its success.
Finally, the Editor-in-Chief thanks Elsevier Science Publishers B.V. for the efforts undertaken so far and to congratulate all persons involved for their courageous decision to engage in this project.


\section{Mini-curriculum vitae of Peter F. Niederer}

Peter F. Niederer was born in 1941 and graduated from the University of Zurich in Theoretical Physics in 1967. He obtained his Ph.D. in Engineering Mechanics from the Swiss Federal Institute of Technology in Zurich, Switzerland in 1972. $\mathrm{He}$ then joined the Institute of Biomedical Engineering and Medical Informatics of the University of Zurich and the Swiss Federal Institute of Technology, from where he left in 1973 to work at the Biomedical Department of General Motors Research Laboratories in Warren, Michigan. Later on, he returned to the Institute of Biomedical Engineering and Medical Informatics. In spring 1980 he was a Visiting Faculty Member at the Department of Mechanical Engineering of the University of Houston, Texas. At present he is Full Professor of Biomedical Engineering at the Swiss Federal Institute of Technology in Zurich. 